\title{
A Hybrid Encryption Technique for Digital Holography using DCT and DWT
}

\author{
Hyun-Jun Choi, Young-Ho Seo, and Dong-Wook Kim, Member, KIMICS
}

\begin{abstract}
In this paper, we present a hybrid encryption for a digital hologram which is the most valuable image content. The encryption algorithm is based on a hybrid technique implementation a fourdimensional transform combining the discrete wavelet transform(DWT) and the discrete cosine transform (DCT). The encryption scheme is composed on the basis of the energy distribution. The experimental results showed that encrypting only $0.0244 \%$ of the entire data was enough to hide the constants of the hologram. The encryption algorithm expected to be used effectively on the researches on encryption and others for digital holographic display.
\end{abstract}

Index Terms - Digital Holography, Image Security, Encryption, DCT/DWT

\section{INTRODUCTION}

CURRENT multimedia era should satisfy the users' demands for more information-implicative and multimedia data services. These have made the communication of digital data very common and much of them retains the very privacy or contents on business, which in turn, needs to hide the contents themselves from the unauthorized persons.

Digital hologram is a technique that the interference patterns between the reference light wave and the object light wave is captured with a charge coupled device (CCD) camera or calculated from an algorithm on computer (Computer Generated Hologram, CGH [1]) instead of writing it on a holographic film [2-4]. The original image can be reconstructed by loading the digital hologram on a Spatial Light Modulator (SLM) and illuminating the reference light that is the same as was recording. A hologram is a relatively much expensive 3dimensional image content that, recently, researchers in many institutions around the world are studying encryption techniques for holograms. But most of them are the optical methods which use optical elements or optical parameters to hide the information [5-11].

Manuscript received March 21, 2011; revised April 3, 2011; accepted April 14, 2011.

Hyun-Jun Choi is with Department of Information and Communication Engineering, Anyang University, 708-113, Anyang 5dong, Manan-gu, Gyeonggi-do, 430-714, Korea (Email: hjchoi@, anyang.ac.kr)
In this paper, we try to encrypt a digital hologram electronically, not optically. This encryption algorithm is based on a hybrid technique implementing a dual transform combing DCT (Discrete Cosine Transform) [12] and DWT (Discrete Wavelet Transform) [13]. In each domain, we try to find the best encryption method. Therefore, the main propose of this paper is to examine the possibility of electronic encryption of a digital hologram. For the digital hologram, we use a digital hologram created by means of the CGH technique. For the data-scrambling scheme we use the standard secretkey encryption algorithm such as 3DES/ASE, etc. even though other schemes are possible.

This paper is organized as follows. The proposed hybrid encryption algorithm is described in Section II. In Section III, the performance of this technique is compared with previous encryption technique. Finally, the conclusion will be given in Section IV.

\section{HYBRID ENCYPTION ALGORITHM}

In this section, we explain the digital holography and then encryption and decryption technique for digital holograms is proposed.

\section{A. Digital Holography and encryption}

In general, a digital hologram is acquired by capturing the phase of interference pattern between the reference light and the object light reflected from an object with CCD camera, and recorded in digital format, as shown in Fig. 1. Therefore, a digital hologram shows substantially different characteristics from a natural image which shows self-similarity that becomes the foundation of fractal theory and strong correlation between adjacent pixels in an image[14].

The use of CCD camera in the acquisition process of a digital hologram inherently includes some noises because of the environments of camera, which dramatically decreases the quality of reconstructed images with the digital hologram.

The proposed encryption process is simply shown in Fig. 1. The diffraction pattern that is encrypted by applying the encryption algorithm on the phase difference between the reflected and reference waveform is obtained. A digital hologram is saved and transmitted in the encrypted state. If it is reproduced without decryption 


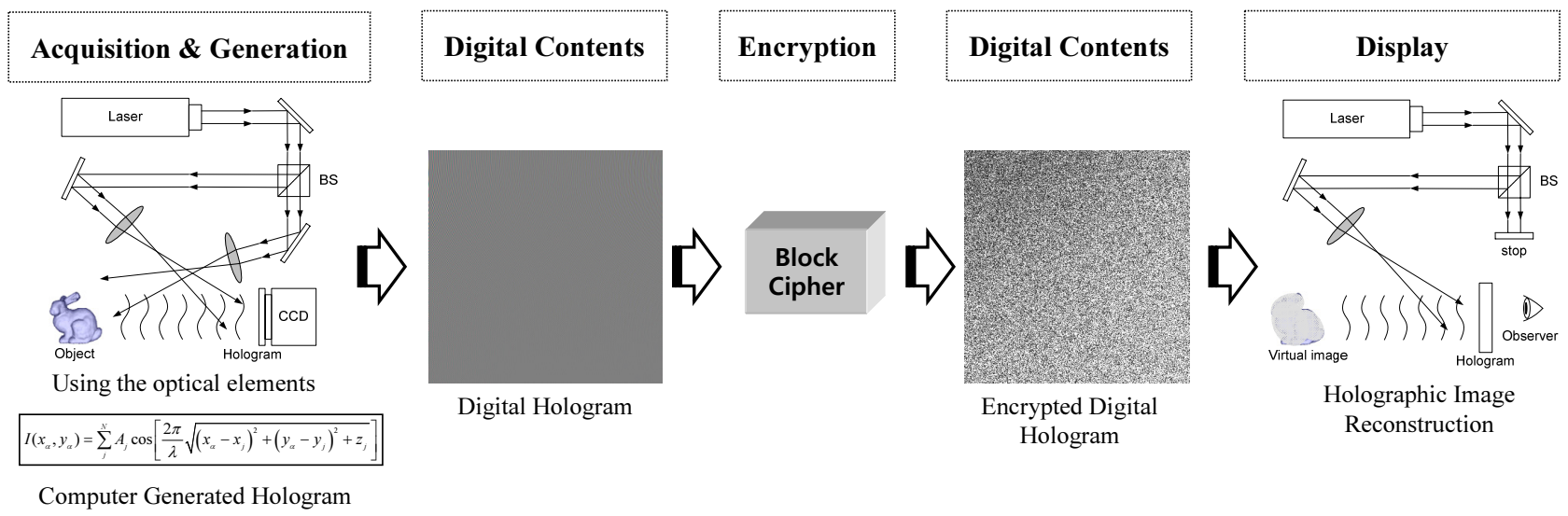

Fig. 1. Concept of digital hologram encryption

process, a distorted object is observed, as shown in Fig. 1.

\section{B. Hybrid encryption algorithm}

This encryption algorithm is based on a hybrid technique implementing a dual transform combing DCT and DWT. For encryption, 2D transform (DCT and DWT) is performed by regarding a digital hologram as a natural 2D image. 2DDCT is the base transform of JPEG and MPEG techniques, while 2DDWT is used in JPEG2000 and MPEG-4 Part10 AMD1 SE SVC. For DCT, we examine the various sizes of transform block. That is, various segmentations with different segment sizes are considered. Once a fringe pattern is segmented, each segment is going to be the unit of 2DDCT (Global DCT, GDCT).

For DWT, Mallat-tree DWT (2DDWT) is used, which is the most general. By DWT, data signals are decomposed to concentrate the energy to lower frequency subbands. Thus, we will examine how much energy needs to be scrambled to hide the information of the holographic image.

If we analyze the results shown in the properties about sampling, localization, and transform, we can obtain two characteristics [14]. First is that the local region of a digital hologram has the information about the entire object. Second id that it has a frequency characteristic that is different from natural images. From such characteristics, the following conclusions can be made. Because the local region of a digital hologram includes information for the entire object, we must encrypt the entire hologram rather than the segmented hologram. DWT that processes the entire 2D information is more appropriate compared to DCT which partitions and transforms the $2 \mathrm{D}$ data. While it is possible to apply DCT for the entire 2D data, the global DCT for the entire information cannot be used in practice because of the enormous amount of the required calculation.
The DCT and DWT transformations were integrated together in a hybrid architecture in a way which enables us to exploit the energy distribution.

In the first step, a DCT is applied to each digital hologram. The DCT was successfully applied to digital hologram encryption [11]. In the next step, called "merging", DC coefficients belonging to different segmentation images are grouped into blocks which are DWT'ed. Finally, some of the subbands are encrypted by a block cipher scheme (here, we used ARIA scheme, but other block cipher methods showed similar results). The proposed algorithm process is shown in Fig. 1. The first algorithmic step in the hybrid algorithm divides the digital hologram $H(x, y)$ into rectangular tiles on a regular grid of the segmented hologram $h_{m, n}(x, y)$. A 2DDCT is performed on each tile $h_{m, n}(x, y)$, with respect to $\mathrm{m}$ and $\mathrm{n}$. The following step in our encryption algorithm is the merging stage. With merging, we implement a splitmerge method with which DC coefficients are rearranged to form a set of input data. The process of merging is shown in Fig. 1. The obtained image (DC coefficients) is then 2DDWT'ed in the next step. As can see in the previous research [11], the result from encrypting LH subband shows the best efficiency. By considering the fact mentioned previously and examining various cases, we decided to choose the subband combinations to be encrypted as follow, which is on the assumption that 6-level MDWT is performed. The encryption methodology is the following:

\section{Forming encryption blocks \\ 2. Encrypting each block \\ 3. Re-located the subbands \\ 4. Inverse MDWT \\ 5. Reconstruction}




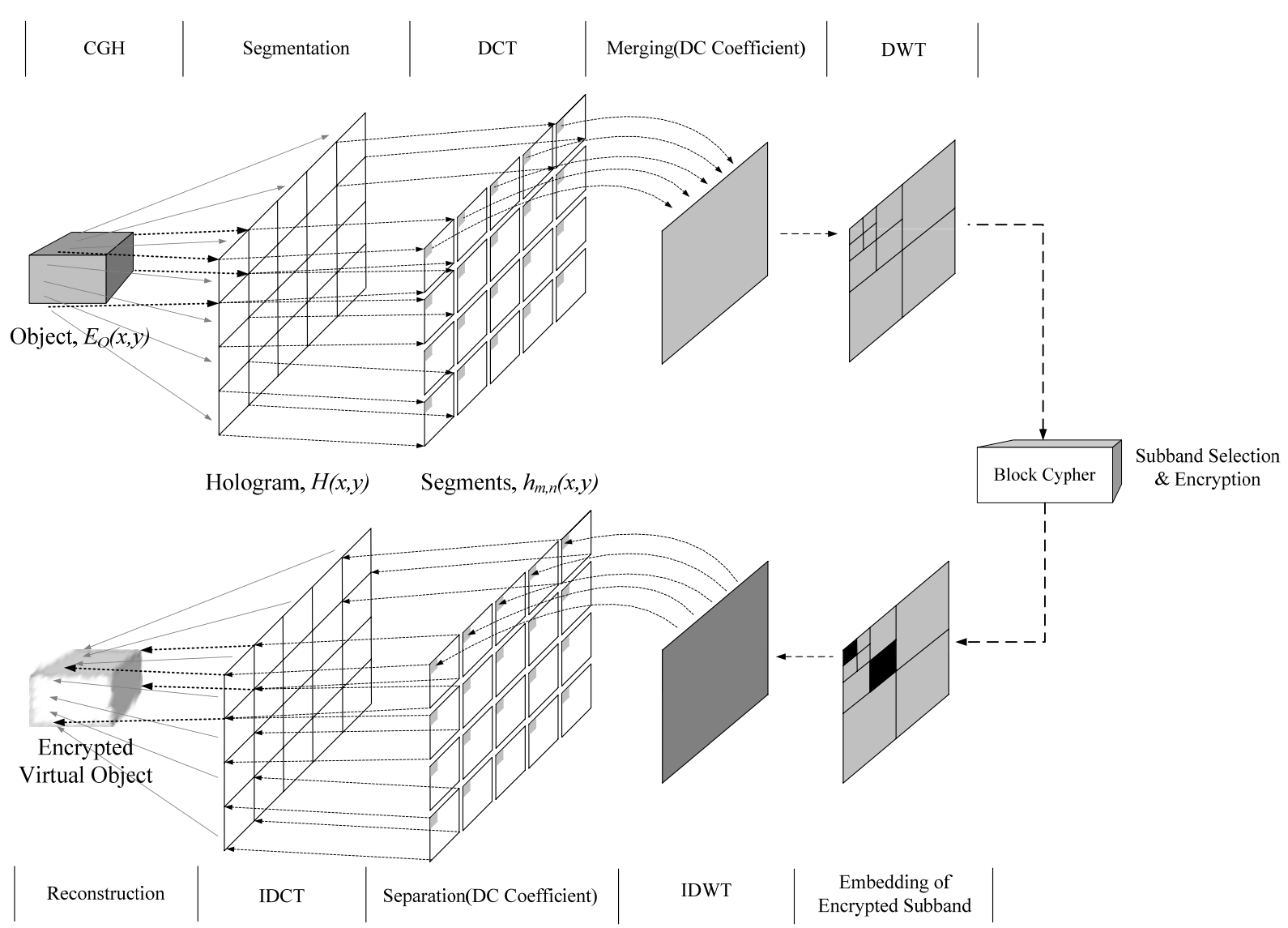

Fig. 2. Hybrid encryption scheme

\section{EXPERIMENTAL RESULTS}

In this section, the proposed encryption technique is applied to digital holograms to examine the encryption effect.

\section{A. Experimental set-up}

To apply the proposed algorithm, digital holograms of 3D object (depth-map, intensity) are generated using the CGH method. The proposed algorithm is applied to them. The encryption results are verified numerically using peak noise-to-signal ratio (PSNR) and normalized correlation (NC). In addition to the numerical statistics, visual observation is used to determine the encryption efficiency.

Experimental environments set up for PC simulation and optical reconstruction are shown in Table I. The size of the digital hologram is $1024 \times 1024\left[\right.$ pixel $\left.^{2}\right]$.

\section{B. Encryption effect}

From this section, the experimental results from applying the proposed algorithm are presented with the values of NC, PSNR and subjective visual analysis.

Table II is to compare the proposed method with two previous hologram encryption methods [11], which used digital signal processing methods. The first method to be compared is the using DCT that scrambled the DCT coefficients of DCTed hologram. The next method is the previous work by the same authors as this paper and was using conventional DWT. The proposed method and previous method are same encrypts ratio, but the PSNR and $\mathrm{NC}$ value are much lower.

TABLE I

EXPERIMENTAL SETUP

\begin{tabular}{|c|c|c|c|}
\hline \multicolumn{2}{|r|}{ Item } & $\mathrm{PC}$ & Optical System \\
\hline \multirow{2}{*}{$\begin{array}{l}\text { SL } \\
\text { M }\end{array}$} & $\begin{array}{c}\text { Resolution } \\
{\left[\text { pixel }^{2} \text { ] }\right.}\end{array}$ & $1024 \times 1024$ & $1024 \times 1024$ \\
\hline & $\begin{array}{l}\text { Pixel pitch } \\
\text { [um] }\end{array}$ & 10.4 & 13.62 \\
\hline \multicolumn{2}{|c|}{ Wavelength [nm] } & 633 & 532 \\
\hline \multicolumn{2}{|c|}{$\begin{array}{c}\text { Reconstruction } \\
\text { distance }[\mathrm{cm}]\end{array}$} & 1,000 & 1,100 \\
\hline
\end{tabular}

The encrypted results are depicted in Fig. 3. From the figure, it can be figured out that at least LL4 should be encrypted to hide the image information unrecognizably. The amount of data of LL4 is one $256^{\text {th }}$ of that of original digital hologram, which is $64 \times 64$ 
[pixel ${ }^{2}$ ] in size and about 32,768 bits if the size of digital hologram is $1,024 \times 1,024\left[\right.$ pixel $\left.^{2}\right]$ and a coefficient has 8 bits.

First, the effect of encryption of proposed algorithm was examined. That is, the data after inverse DCT with reconstruction as Fig. 3 are examined. NC and PSNR were measured for the data before and after encryption and shown in Table II. If NC and PSNR results are examined closely, it is possible to arrive at the conclusion that it is difficult to accurately determined the encryption effect with just numerical results. Therefore, we examined the effect with visual observation as well as the numerical values of NC and PSNR.

TABLE II

ENCRYPTION RESULTS

\begin{tabular}{|c|c|c|c|c|c|}
\hline \multicolumn{2}{|c|}{ Methods } & $\begin{array}{c}\text { \# of bits } \\
\text { encryption }\end{array}$ & $\begin{array}{c}\text { Encryption } \\
\text { ratio }\end{array}$ & $\begin{array}{c}\text { Average } \\
\text { PSNR }\end{array}$ & $\begin{array}{c}\text { Average } \\
\text { NC }\end{array}$ \\
\hline \multirow{2}{*}[11]{} & DCT & 3,584 & 0.0244 & 8.75 & 0.45 \\
\cline { 2 - 6 } & DWT & 2,048 & 0.0244 & 11.25 & 0.71 \\
\hline \multicolumn{2}{|c|}{ This paper } & 2,048 & 0.0244 & 8.51 & 0.41 \\
\hline
\end{tabular}

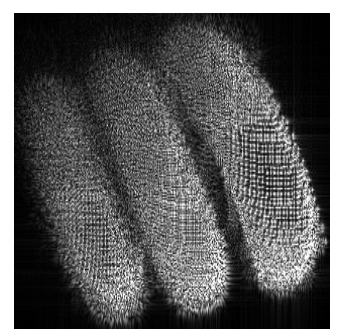

(a)

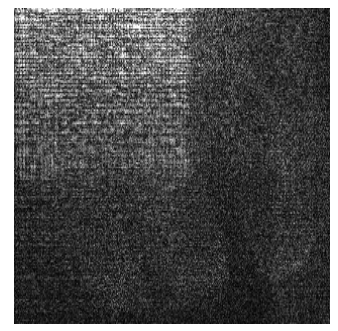

(b)

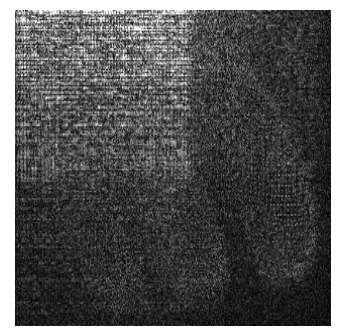

(b)

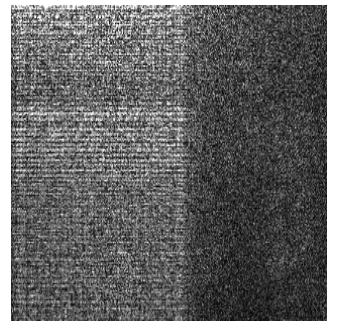

(d)

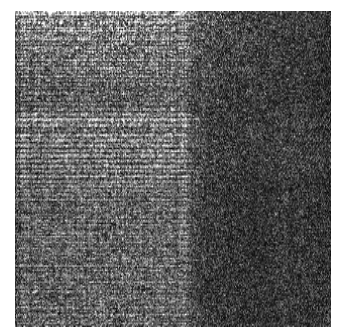

(d)

Fig. 3. The reconstructed holographic image after encrypting for spring; (a) no subband, (b) LL2, (c) LL2-HH2, (d) LL1, (e) LL1-LH1.

\section{CONCLUSIONS}

This paper proposed a hybrid encryption technique implementing a dual transform combining DCT and DWT. We included the schemes two frequency-domain (DCT and DWT). If a strong encryption scheme is needed and all the processing cost is devoted to encryption, the scheme to encrypt more hologram data.

The hybrid encryption scheme has shown significantly better results compared with the previous encryption schemes[11].

It is hoped that the digital hologram encryption technique by this paper would become a useful guide for those who are researching or will research this area.

\section{ACKNOWLEDGMENT}

This work was supported by the National Research Foundation of Korea Grant funded by the Korean Government(MEST) (NRF-2010-0022080)

\section{REFERENCES}

[1] B. R. Brown and A. W. Lohmann, "Computer-generated Binary Holograms," IBM Journal of research and Development, Vol. 13, No. 2, pp.160-168, March 1969.

[2] B. Javidi and F. Okano, "Three Dimensional Television, Video, and Display Technologies," Springer Verlag Berlin. March 2002.

[3] P. Hariharan, "Basics of Holography," Cambridge University Press, 2002.

[4] H. Yosikawa, "Digital holographic signal processing," Proc. TAO First International Symposium on Three Dimensional Image Communication Technologies, 1993.

[5] X. Wang, D. Zhao, and L. Chen, "Image encryption based on extended fractional Fourier transform and digital holography technique," Optics Communication, Vol. 260, pp. 449-453, 2006.

[6] L. Chen and D. Zhao, "Optical color image encryption by wavelength multiplexing and lensless Fresnel transform holograms," Optics Express, Vol. 14, No. 19, Sept. 2006.

[7] Y.-Y. Wang, Y.-R. Wang, Y. Wang, H.-J. Li, and W.-J. Sun, "Optical image encryption based on binary Fourier transform computer-generated hologram and pixel scrambling technology," Optics and Lasers Eng., Vol. 45, pp. 761-765, 2007.

[8] E. Tajahuerce and B. Javidi, "Encryption three-dimensional information with digital holography," Appl. Opt. Vol. 39, pp. 6595-6601, Dec. 2000.

[9] H. Kim, D.-H. Kim, and Y. H. Lee, "Encryption of digital hologram of 3-D object by virtual optics," Optics Express, Vol. 12, No. 20, pp. 4912-4921, Oct. 2004.

[10] T. J. Naughton and B. Javidi, "Compression of encrypted threedimensional objects using digital holography," Opt. Eng., Vol. 43, No. 10, pp. 2233-2238, Oct. 2004.

[11] D.-W. Kim, H.-J. Choi, Y.-G. Choi, J.-S. Yoo, and Y.-H. Seo, "Information hiding for digital holograms by electronic partial encryption methods," Optics communication, Vol. 277, Issue 2, pp. 277 287, Sept. 2007.

[12] R. C. Gonzalez and R. E. Woods, Digital Image Processing, 3rd Edition, Pearson Prentice Hall, 2009, pp. 291-298.

[13] R. M. Rao, A. S. Bopardikar, and T. Boros, "Wavelet transforms, introduction to theory and application," Prentice Hall PTR, 1st edition, Sept. 1998.

[14] Y.-H. Seo, H.-J. Choi, and D.-W. Kim, "Lossy Coding Technique for Digital Holographic Signal", SPIE Optical Engineering, Vol. 45, No. 6, pp. 065802-1 065802-10, Jun. 2006. 


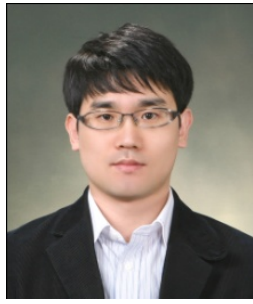

Hyun-Jun Choi has received his M.S. and Ph.D. degrees in 2005 and 2009 from Dept. of Electronic Materials Engineering of Kwangwoon University in Seoul, Korea. He was a research professor in Realistic Media Institute at Kwangwoon University. $\mathrm{He}$ is currently an assistant professor with the department of Information and Communication Engineering, Anyang University, Anyang-si, Korea. He is research interests are in optical image processing and 3D display.

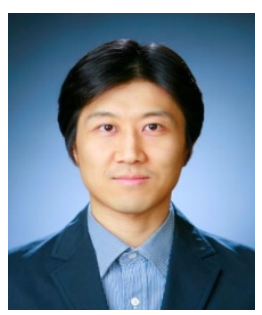

Young-Ho Seo has received his M.S and Ph.D degree in 2000 and 2004 from Dept. of Electronic Materials Engineering of Kwangwoon University in Seoul, Korea. He was a researcher at Korea Electrotechnology Research Institute (KERI) in 2003 to 2004. He was a research professor in Dept. of Electronic and Information Engineering at Yuhan College in Buchon, Korea in 2005. He was an professor of Dept. of Information and Communication Engineering at Hansung University in Seoul, Korea in 2006 to 2007. He is now an assistant professor of College of Liberal Arts at Kwangwoon University in Seoul, Korea and a director of research institute in Ten Technology Inc. His research interests include 2D/3D digital image processing, SoC design and contents security

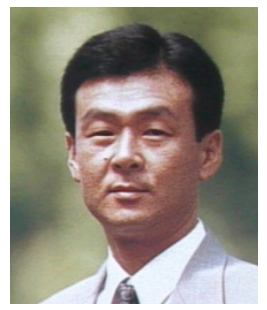

Dong-Wook Kim (S'82-M'85) received the B.S. and M.S. degrees from the Department of Electronic Engineering, Hangyang University, Seoul, Korea, in 1983 and 1985, respectively, and the Ph.D. degree from the Department of Electrical Engineering, Georgia Institute of Technology, Atlanta, in 1991. He is currently a Professor and the Dean of Academic Affairs at Kwangwoon University, Seoul. His current research interests include digital system design, digital testability and design-for-test, digital embedded systems for wired and wireless communication, and design of digital signal processors. 